This article was published as "O. Alijevic, H. Hammoud, A. Vaithia, V. Trendafilov, M. Bollenbach, M. Schmitt, F. Bihel, S. Kellenberger, Heteroarylguanidines as Allosteric Modulators of ASIC1a and ASIC3 Channels, ACS chemical neuroscience 9(6) (2018) 1357-1365, doi: 10.1021/acschemneuro.7b00529", and can be found at: https:// pubs.acs.org/doi/10.1021/acschemneuro.7b00529

\title{
Heteroarylguanidines as Allosteric Modulators of ASIC1a and ASIC3 Channels
}

Omar Alijevic ${ }^{1}$, Hassan Hammoud ${ }^{2}$, Anand Vaithia ${ }^{1}$, Viktor Trendafilov ${ }^{1}$, Maud Bollenbach ${ }^{2}$, Martine Schmitt $^{2}$, Frédéric Bihel ${ }^{2 *}$, Stephan Kellenberger ${ }^{1 *}$

${ }^{1}$ University of Lausanne, Department of Pharmacology and Toxicology, 1011 Lausanne, Switzerland.

${ }^{2}$ University of Strasbourg, Faculty of Pharmacy, 67401 Illkirch Cedex, France

Running title: Modulation of ASICs by GMQ derivatives

Keywords: acid-sensing ion channel, channel regulation, structure-activity relationship, compound testing

*Contact information: Concerning functional evaluation and ASICs: Stephan Kellenberger, Department of Pharmacology and Toxicology, Rue du Bugnon 27, University of Lausanne, CH-1011 Lausanne, Switzerland, phone ++4121 6925422, stephan.kellenberger@unil.ch; concerning medicinal chemistry: Frédéric Bihel, Faculty of Pharmacy, 74 Route du Rhin CS60024, University of Strasbourg, F-67401 Illkirch - Cedex, France, phone ++33 368854130, fbihel@unistra.fr 


\section{Abstract}

Acid-sensing ion channels (ASICs) are neuronal $\mathrm{Na}^{+}$-selective ion channels that open in response to extracellular acidification. They are involved in pain, fear, learning and neurodegeneration after ischemic stroke. 2-Guanidine-4-methylquinazoline (GMQ) was recently discovered as the first nonproton activator of ASIC3. GMQ is of interest as a gating modifier and pore blocker of ASICs. It has however a low potency, and exerts opposite effects on ASIC1a and ASIC3. To further explore the molecular mechanisms of GMQ action, we have used the guanidinium moiety of GMQ as a scaffold and tested the effects of different GMQ derivatives on the ASIC pH dependence and maximal current. We report that GMQ derivatives containing quinazoline and quinoline induced, as GMQ, an alkaline shift of the $\mathrm{pH}$ dependence of activation in ASIC3 and an acidic shift in ASIC1a. Another group of 2-guanidinopyridines shifted the pH dependence of both ASIC1a and ASIC3 to more acidic values. Several compounds induced an alkaline shift of the pH dependence of ASIC1a/2a and ASIC2a/3 heteromers. Compared to GMQ, guanidinopyridines showed a 20 -fold decrease in the $\mathrm{IC}_{50}$ for ASIC1a and ASIC3 current inhibition at pH5. Strikingly, 2-guanidino-quinolines and -pyridines showed a concentrationdependent biphasic effect that resulted at higher concentrations in ASIC1 a and ASIC3 inhibition $\left(\mathrm{IC}_{50}>100 \mu \mathrm{M}\right)$, while causing at lower concentration a potentiation of ASIC1a, but not ASIC3 currents $\left(\mathrm{EC}_{50} \approx 10 \mu \mathrm{M}\right)$. In conclusion, we describe a new family of small molecules as ASIC ligands and identify an ASIC subtype-specific potentiation by a subgroup of these compounds.

Acid-sensing ion channels (ASICs) are $\mathrm{Na}^{+}$-selective ion channels that are activated by a rapid drop in extracellular $\mathrm{pH}^{1,2}$. They form a sub-family of the $\mathrm{ENaC} /$ degenerin channel superfamily, to which besides ASICs the Epithelial $\mathrm{Na}^{+}$channel $\mathrm{ENaC}$, the C. elegans degenerins and the mollusk channel FaNaC belong ${ }^{1}$. ENaC plays a role in transepithelial $\mathrm{Na}^{+}$transport, the degenerins are part of a mechanotransduction complex, while $\mathrm{FaNaC}$ is a peptide-gated neuronal ion channel. ASICs are mostly found in the nervous system, and their activation induces a membrane depolarization of the neurons in which they are expressed, affecting thereby neuronal signaling ${ }^{3,4}$. The subunits ASIC1a, - 
$1 \mathrm{~b},-2 \mathrm{a},-2 \mathrm{~b}$ and -3 can assemble into homo- or heterotrimeric channels ${ }^{5,6}$. Biophysical properties of the ASICs, such as $\mathrm{pH}$ dependence and current kinetics, depend on the subunit composition, and it has been shown that ASIC1a is especially important in the central nervous system, while ASIC3 appears to be the most important ASIC subunit in the peripheral nervous system ${ }^{1,7}$. Studies with ASIC knockout mice provided evidence for roles of ASICs in learning, fear behavior, neurodegeneration after ischemic stroke, mechanosensation and pain sensation ${ }^{1,7-14}$. ASICs are therefore attractive potential targets for analgesic and anxiolytic drugs and for the pharmacological treatment of stroke. Positive allosteric modulators of ASICs may improve learning. The prototype ASIC inhibitor amiloride is used as a $\mathrm{K}^{+}$sparing diuretic due to its high affinity inhibition of $\mathrm{ENaC}^{15,16}$. On ASICs, amiloride has however a low potency $\left(\mathrm{IC}_{50}\right.$ of 10-100 $\mu \mathrm{M}$, compared to $100 \mathrm{nM}$ on $\mathrm{ENaC}^{l}$ ). Amiloride concentrations required to inhibit ASIC currents affect also other transporters and ion channels ${ }^{17}$. In spite of efforts of several laboratories and drug companies, it seems that so far no clearly superior small molecule ASIC inhibitors have been discovered ${ }^{1,7,18-22}$. In contrast, several toxins inhibit ASIC currents with nanomolar affinity 23.

For many years, protons were the only known activators of ASICs. In the search for other ASIC activators, a large number of ASIC modulators has been identified ${ }^{1,7}$. Such modulators, which are for example divalent and polyvalent ions, small molecules, and peptides, change in most cases the ASIC $\mathrm{pH}$ dependence ${ }^{1,7}$. Recently, the molecule 2-guanidine-4-methylquinazoline (GMQ) was shown to activate ASIC3 at physiological pH7.4 and to induce pain in an ASIC3-dependent manner, when injected into the paw of a mouse ${ }^{24}$. Our laboratory has subsequently shown that GMQ induces ASIC3 activation by changes in its $\mathrm{pH}$ dependence that create a window current at $\mathrm{pH}$ 7.4. The GMQ-induced changes in ASIC3 $\mathrm{pH}$ dependence are different from the gating modulation by GMQ observed in other ASIC subtypes ${ }^{25}$. GMQ can activate the ENaC/degenerin family member FaNaC, but has no effect on $\mathrm{ENaC}$ currents ${ }^{26}$. It has also been shown that the endogenous arginine metabolites agmatine and arcaine exert similar effects on ASIC3 currents ${ }^{27}$ as does GMQ. It is therefore possible that GMQ-like substances may be endogenous modulators of ASICs. GMQ contains, like amiloride, a guanidinium group. Interestingly, it was observed that GMQ inhibits ASICs by a pore block in addition to its effects on gating ${ }^{25}$, and that at high concentrations, amiloride can affect the ASIC3 gating in a similar way as 
does GMQ ${ }^{28,29}$. It would be interesting to better understand the molecular mechanisms of GMQ action and to use GMQ as a pharmacological tool in animal and cellular studies. Currently, the use of GMQ is limited by its low potency, with $\mathrm{EC}_{50}$ and $\mathrm{IC}_{50}$ values of the order of $2 \mathrm{mM}^{24,25}$. The discovery of GMQ opened however the way for the design of new chemical entities modulating the dependence of ASIC activity on $\mathrm{pH}$.

The aim of the present study was to find GMQ analogs with improved affinity, and to understand the structure-activity relationship of GMQ-like compounds on ASICs. Among the derivatives tested, we identified groups with distinct effects on ASIC $\mathrm{pH}$ dependence. Most of the compounds showed also a strong inhibition of the maximal peak current of ASIC1a, but less of ASIC3. In ASIC1a/2a and ASIC2a/3 heteromers, the maximal current inhibition by these compounds was less pronounced, and shifts in $\mathrm{pH}$ dependence, if they occurred, were similar to those observed with ASIC3. Several compounds exerted a biphasic action on ASIC1a, inducing potentiation at concentrations of 10-80 $\mu \mathrm{M}$, and inhibition at $\geq 100 \mu \mathrm{M}$.

\section{Results and Discussion}

\section{Compound library and testing protocol}

ASIC activation by acidification leads only to a transient channel opening, because these channels desensitize rapidly (within hundreds of ms to s) after activation ${ }^{l}$. ASICs can exist in three different functional states, closed, open and desensitized. The $\mathrm{pH}$ dependence of activation (Fig. 1a), the transition from the closed to the open state, determines the current induced by a solution change from $\mathrm{pH} 7.4$ to a given acidic $\mathrm{pH}$. The $\mathrm{pH}$ that induces half of the maximal current amplitude $\left(\mathrm{pH}_{50}\right)$ is 6.56.7 for ASIC1a and ASIC3 ${ }^{30,31}$. When ASICs are exposed for tens of seconds to a pH that is more acidic than $\mathrm{pH} 7.4$, but not sufficiently acidic to open them, they can enter the desensitized state without apparent opening, in a process called steady-state desensitization (SSD). The midpoint of SSD $\left(\mathrm{pHD}_{50}\right)$ is $\sim 7.2$ for ASIC1a and $\sim 7.1$ for ASIC $3{ }^{1}$. The $\mathrm{pH}$ dependence of SSD determines the availability of the channels for opening at a given basal $\mathrm{pH}$. It was previously shown that GMQ shifts the $\mathrm{pH}$ dependence of activation of ASIC1a to more acidic, and that of ASIC3 to more alkaline values, as illustrated 
schematically in Fig. 1a ${ }^{25,29}$. Due to an additional acidic shift of the pH dependence of SSD in ASIC3, GMQ induces a sustained (i.e. a non- or partially desensitizing) current at $\mathrm{pH} \geq 6$ in this ASIC subtype ${ }^{25}$. In addition, GMQ decreases the maximal current amplitude induced by acidification to $\mathrm{pH} 5$ due to its pore-blocking effect (Fig. 1a) $)^{25}$. To characterize the effects of GMQ derivatives, the current response was measured at pH6.6 to detect effects on $\mathrm{pH}$ dependence ("gating effect"), and at $\mathrm{pH}$, where the current is maximal, to quantify the pore block ("blocking effect"), as illustrated in Fig.1a with the hypothetical $\mathrm{pH}$ dependence curves of ASIC1a and ASIC3 in the absence or presence of a GMQ derivative. Experiments were carried out with $\mathrm{CHO}$ cell lines stably expressing human ASIC1a ${ }^{32}$ or rat ASIC $3{ }^{30}$, at compound concentrations of 0.3 and $1 \mathrm{mM}$. Typical current traces obtained under such conditions in the absence and presence of the GMQ derivatives 5a in ASIC1a and 7, as well as $\mathbf{4 a}$ in ASIC3, are shown in Fig. 1b. A sustained current was induced by compound 4a (Fig.1b, bottom panel; the compounds used in this study are shown in Fig. 2). The ASIC3 current increase at pH6.6 in the presence of compound 7 (Fig. 1b, middle panel) indicates an alkaline shift in $\mathrm{pH}$ dependence. An inhibition of the pH5-induced current was observed with all three compounds shown in Fig. 1b.

The initial analysis of the structure-activity relationship of GMQ by Yu et al. ${ }^{24}$ was limited to the 2guanidino-4-methylquinazoline scaffold, since 2-guanidino-benzimidazole, -benzothiazole and benzoxazole were found to modulate ASIC3 poorly or not at all. In the present study, we modified the quinazoline scaffold of GMQ (Fig. 2). In a first series (cluster \#1), we conserved the bicyclic system of GMQ, but we replaced the benzene ring by a cyclohexene ring (1) to evaluate the influence of the aromatic ring on activity. We also synthesized quinoxaline (2) and quinoline (3a, b) bicycles as GMQ analogs, in order to evaluate the role of the methyl group at position 4, but more importantly because the removal of the nitrogen atom at position 3 of GMQ limits the formation of an H-bond interaction with guanidine to the nitrogen atom at position 1. In a second series, we disconnected both rings to generate the 6, 5, and 4-phenyl-2guanidinopyridines, forming clusters \#2 (4a-c), \#3 (5a-e), and \#4 (6), respectively. Finally, we investigated the loss of the guanidine moiety with the 2-aminopyridines 7 and 8 (cluster \#5).

Blocking effect of GMQ and its derivatives on ASICla at pH5 
At $\mathrm{pH} 5$ and a concentration of $1 \mathrm{mM}$, GMQ inhibited the maximal current of ASICla $\left(I_{\mathrm{GMQ}} / I_{\mathrm{ctrl}}=0.68\right.$ \pm 0.06 at $\mathrm{pH} 5 ; \mathrm{n}=7, \mathrm{p}<0.01 ;$ Fig.3a, filled bars $)$. Compound 1 showed no significant inhibition $\left(I_{\text {cpd }} / I_{\text {ctrl }}\right.$ $=0.81 \pm 0.10, \mathrm{p}>0.05$ ), while quinoxaline $\mathbf{2}$, as well as the quinoline $\mathbf{3} \mathbf{b}$, showed a similar modest inhibition as GMQ. Quinoline 3a, however, produced a stronger inhibition than GMQ $\left(I_{\text {cpd }} / I_{\text {ctrl }}=0.26\right.$ $\pm 0.13, \mathrm{p}<0.01 \mathrm{vs}$. GMQ). This suggests that the nitrogen atom at position 3 of GMQ alters the blocking effect on ASIC1a. Interestingly, all the guanidinopyridine analogs (clusters \#2, \#3, and \#4) showed a blocking effect on ASIC1a that was similar or even greater than that by GMQ. While the presence of an aryl group at position 6 of the pyridine did not change the blocking effect (4a-c), aryl groups at position $3(\mathbf{5 b}-\mathbf{c})$ or $4(\mathbf{6})$ led to efficient pore blockers with $I_{\text {cpd }} / I_{\text {ctrl }}$ ratios of $0.24 \pm 0.08(\mathrm{p}<0.01), 0.16$ $\pm 0.11(\mathrm{p}<0.0001)$, and $0.22 \pm 0.02(\mathrm{p}<0.001)$, respectively. Finally, the two compounds lacking the guanidine moiety, 5-phenyl-2-aminopyridine (7) and 6-phenethyl-2-aminopyridine (8) showed a moderate inhibition quite similar to the one by GMQ.

\section{Gating effect of GMQ and its derivatives on ASICla at pH6.6}

The $\mathrm{pH}_{50}$ of ASIC1a and ASIC3 activation is $\sim 6.5$ and $\sim 6.7$, respectively ${ }^{1,7,25}$. Modulatory effects of test compounds on the ASIC pH dependence were measured at pH6.6 (Fig. 3a, open bars). Because pH6.6 is within the steep range of the $\mathrm{pH}$ - current relationship (Fig. 1a), the pH6.6-induced current is very sensitive to changes in $\mathrm{pH}$ dependence. GMQ at $1 \mathrm{mM}$ abolished the $\mathrm{pH} 6.6$-induced current of ASIC1a ("gating effect", $I_{\mathrm{GMQ}} / I_{\mathrm{ctrl}}=0.03 \pm 0.01$ ), while reducing the $\mathrm{pH}$ 5-induced peak amplitude ("blocking effect") by $\sim 30 \%$. With compounds that induce an inhibition of the maximal current amplitude (filled bars in Fig. 3a), an $\mathrm{I}_{\mathrm{cpd}} / \mathrm{I}_{\mathrm{ctrl}}$ ratio at pH6.6 (open bars) that is smaller than the corresponding ratio at $\mathrm{pH} 5$ indicates an acidic shift of the $\mathrm{pH}$ dependence due to the tested compound, while an $\mathrm{I}_{\mathrm{cpd}} / \mathrm{I}_{\mathrm{ctrl}}$ ratio at $\mathrm{pH} 6.6$ higher than that at $\mathrm{pH} 5$ indicates an alkaline shift. Based on these ratios at $\mathrm{pH} 5$ and $\mathrm{pH} 6.6$, and under the assumption that the compounds did not change the steepness of the $\mathrm{pH}$ - current relationship, we estimated the shift of $\mathrm{pH}_{50}\left(\Delta \mathrm{pH}_{50}\right)$ for the activation curve of each compound in comparison with the control condition (Fig $3 \mathrm{~b},\left(\Delta \mathrm{pH}_{50}>0\right.$ : alkaline shift; $\Delta \mathrm{pH}_{50}<0$ : acidic shift; see Methods). Fig. $3 \mathrm{~b}$ indicates thus the estimated shifts in $\mathrm{pH}$ dependence induced by the different compounds.

GMQ and its analogs, except for $\mathbf{4 a - b}, \mathbf{6}$ and 7, produced $\mathrm{I}_{\mathrm{cpd}} / \mathrm{I}_{\mathrm{ctrl}}$ ratios at $\mathrm{pH} 6.6$ that were significantly different from the $\mathrm{I}_{\mathrm{cpd}} / \mathrm{I}_{\mathrm{ctrl}}$ ratio measured at $\mathrm{pH} 5.0$. Based on this comparison we can conclude that these 
compounds significantly shift the ASIC1a pH dependence. GMQ analogs from cluster \#1 (yellow bars in Fig. 3, bicycles 1, 2, 3a, 3b), as well as cluster \#3 (red bars, 5-substituted pyridines 5a-e) have thus a qualitatively similar profile as GMQ, inducing an acidic shift of the $\mathrm{pH}_{50}$. Of the 6- and 4-substituted pyridines from cluster \#2 (green bars) and \#4 (purple bar) respectively, only $\mathbf{4 c}$ induced a modest shift in $\mathrm{pH}_{50}$, similarly as compounds from cluster \#1 (1-3). The aminopyridine 8 (cluster \#5) was the only compound to produce an alkaline shift in ASIC1a.

\section{Blocking effect of GMQ and its derivatives on ASIC 3 at pH5}

At $\mathrm{pH} 5$ and a compound concentration of $1 \mathrm{mM}$, GMQ did only slightly decrease the maximal peak current of ASIC3 $\left(I_{\mathrm{GMQ}} / I_{\mathrm{ctrl}}=0.83 \pm 0.07, \mathrm{n}=8\right.$; Fig.4a $)$. Indeed, it had previously been shown that higher GMQ concentrations are needed to efficiently inhibit the $\mathrm{pH} 5$-induced ASIC3 current $\left(\mathrm{IC}_{50}=6.74 \pm\right.$ $0.83 \mathrm{mM})^{25}$. Compounds $\mathbf{1}$ and $\mathbf{2}$ showed no inhibitory activity at $1 \mathrm{mM}(\mathrm{p}>0.05)$, whereas both quinolines (3a and 3b) showed a significant blocking effect with $I_{\mathrm{cpd}} / I_{\mathrm{ctrl}}$ ratios of $0.37 \pm 0.03$ and 0.59 \pm 0.03 , respectively $(\mathrm{p}<0.0001$ and $<0.001, \mathrm{n}=4)$. This result indicates that the substitution of the nitrogen atom at position 3 of GMQ alters the blocking effect on ASIC3, while the methyl group at position 4 has no influence. Interestingly, all the guanidinopyridines from clusters \#2, \#3, and \#4, led to efficient inhibition of ASIC3 at $1 \mathrm{mM}$ with $I_{\text {cpd }} / I_{\text {ctrl }}$ ratios ranging from $0.16 \pm 0.02(6, \mathrm{n}=5)$ to 0.44 $\pm 0.05(\mathbf{5 a}, \mathrm{n}=5)$. In contrast, the presence of a phenethyl group at position 5 led to less efficient pore block (5e vs $5 \mathbf{d}$ p $<0.05$ ). Finally, the compounds 7 and 8 , lacking a guanidine moiety (cluster \#5), showed only a modest blocking.

\section{Blocking effects of GMQ derivatives}

The inhibition experiments at $\mathrm{pH} 5$ show that at $1 \mathrm{mM}$, GMQ and its derivatives belonging to cluster \#1 were mostly poor inhibitors of both channels. The quinolines $\mathbf{3 a}$ and $\mathbf{3 b}$ showed stronger inhibition than GMQ on ASIC3, and 3a on ASIC1a, illustrating the contribution of the nitrogen atom at position 3. In addition, most of the guanidinopyridines (clusters \#2-4) led to a substantial inhibition of both channels. Interestingly, this inhibition seems to be independent of the position of the substituents on the pyridine 
ring, or of the pKa of the molecules (Table S1). Finally, 2-aminopyridines (cluster \#5) were poor inhibitors of both channels.

\section{Gating effect of GMQ and its derivatives on ASIC3 at pH6.6}

At $\mathrm{pH} 6.6$ and a concentration of $1 \mathrm{mM}$, GMQ increased the ASIC3 current $\left(I_{\mathrm{GMQ}} / I_{\mathrm{ctrl}}=1.26 \pm 0.09\right.$ at pH 6.6), consistent with a shift of the activation curve to a more alkaline value (Fig 4a). As for ASIC1a, an estimate of the shift in $\mathrm{pH}_{50}$ induced by each of these compounds, based on the peak current ratios at $\mathrm{pH} 5$ and 6.6, is presented in Fig. $4 \mathrm{~b}\left(\Delta \mathrm{pH}_{50}>0\right.$ : alkaline shift; $\Delta \mathrm{pH}_{50}<0$ : acidic shift). Bicyclic analogs from cluster \#1, except quinoxaline 2 , tend to shift the $\mathrm{pH}_{50}$ to alkaline values on ASIC3, in an opposite manner to their shifts on ASIC1a. All 5-substituted guanidinopyridines (5a-e) showed a tendency of, or a significant acidic $\mathrm{pH}_{50}$ shift $(\mathbf{5 b}, \mathbf{5 c}, \mathbf{5 e})$, with $\Delta \mathrm{pH}_{50}$ values ranging from -0.05 to 0.22 . In contrast to compounds of cluster $\# 1$, compounds of cluster $\# 3$ induced an acidic $\mathrm{pH}_{50}$ shift (or a tendency of) in both channels. Finally, both 2 -aminopyridines ( $\mathbf{7}$ and $\mathbf{8})$ induced an alkaline $\mathrm{pH}_{50}$ shift.

\section{Gating effects of $G M Q$ derivatives}

Whereas the position of the substitution on the pyridine ring was not crucial for the inhibitory effect at $\mathrm{pH} 5$, it was clearly important for the shift in $\mathrm{pH}$ dependence. Depending on the position of the aryl group on the pyridine ring, we observed no shift (clusters \#2 and \#4), an alkaline shift in ASIC3 and an acidic shift in ASIC1a (cluster \#1), or a shift to more acidic values in both ASIC subtypes (cluster \#3). This dependence of the gating effect on the position of the aryl group suggests that the position of the aromatic group on the guanidinopyridine may be critical for the interaction with the modulatory GMQ binding site. Finally, the 2-aminopyridine $\mathbf{8}$ was the only tested compound to induce an alkaline shift in ASIC1a.

\section{Do GMQ derivatives induce a sustained ASIC3 current?}

GMQ is known to generate a sustained current in ASIC3 with maximal amplitudes in the $\mathrm{pH}$ range 6.5$7^{25}$. We evaluated the capability of GMQ derivatives to generate a similar effect. The sustained current amplitude, measured at pH6.6 in the presence of $1 \mathrm{mM}$ of the compound, was normalized in two ways (Fig. 4c). First, this sustained current was normalized to the maximal acid-induced current in the 
absence of the compound, as $I_{\text {sust }(\mathrm{pH} 6.6, \mathrm{cpd})} / I_{\text {peak }(\mathrm{pH} 5 \text {, ctrl) }}$ ratio (Fig. $4 \mathrm{~d}$, filled bars). As an indication of the shape of the current, the $I_{\text {sust }(\mathrm{pH} 6.6, \mathrm{cpd})} / I_{\mathrm{peak}(\mathrm{pH} 6.6, \mathrm{cpd})}$ ratio, which compares the amplitude of the sustained current with the amplitude of the peak current induced by the test compound at $\mathrm{pH6.6}$, is provided as open bars in Fig. 4d. For GMQ, these two ratios were similar $(0.36 \pm 0.05$ vs $0.60 \pm 0.02)$, since the pH6.6-induced peak current amplitude in the presence of $1 \mathrm{mM} \mathrm{GMQ}$ is only slightly smaller than the pH5-induced peak current amplitude under control conditions. In the absence of any compound, no sustained current was measured at pH6.6, and both ratios were $0.01 \pm 0.01(\mathrm{n}=16)$. In most derivatives tested, the shape of the current was conserved with regard to GMQ, as indicated by similar $I_{\text {sust } \mathrm{pH} 6.6 \text {, }}$ cpd) $/ I_{\text {peak(pH6.6, cpd) }}$ ratios (open bars). Only the 2-aminopyridines 7 and 8 from cluster \#5 did not induce any sustained current. The $I_{\text {sust }(\mathrm{pH} 6.6, \mathrm{cpd})} / I_{\mathrm{peak}(\mathrm{pH} 5, \text { ctrl) }}$ ratios (filled bars in Fig. $4 \mathrm{~d}$ ) were however considerably smaller than what had been observed with GMQ. The difference in the two ratios is essentially due to the strong current block observed with most GMQ derivatives other than guanidinoquinazolines (Fig. 4a). A substantial $I_{\text {sust(pH6.6, cpd })} / I_{\text {peak(pH5, ctrl) }}$ ratio was only observed with the monocycle 2-guanidino5-phenyl-pyridine 5a and with some compounds of cluster \#1. Together, this suggests that the mechanism underlying the formation of this sustained current is very sensitive to the chemical structure of the modulator, and is also affected by the inherent inhibitory effect of the modulator. In the presence of GMQ, the activation and SSD curves of ASIC3 cross each other at a $\mathrm{pH}$ of $\sim 6.8-7.0$. The $\mathrm{pH}$ dependence of the sustained ASIC3 current amplitude under these conditions forms a bell-shaped curve that has its maximum at the crossing of these two curves, consistent with it being a window current $^{25,29}$. Analysis of the amplitude of the sustained ASIC3 current in the presence of selected GMQ derivatives at several $\mathrm{pH}$ conditions shows maximal sustained current amplitudes at $\mathrm{pH} 7$ or 6.6 (Fig. S1), further supporting that these compounds also induce a window current in ASIC3, as does GMQ. There was no indication of sustained ASIC1a currents in the presence of GMQ derivatives, in agreement with previous observations with GMQ ${ }^{25,29}$.

\section{Concentration dependence of ASIC current inhibition at pH5 by GMQ derivatives}

All experiments described above were carried out at two compound concentrations, 1 and $0.3 \mathrm{mM}$, as documented for the pH5-induced current in Table S2. Comparison of the effects at the two concentrations suggested an increased potency relative to GMQ of some of the test compounds. To confirm the predicted change in $\mathrm{IC}_{50}$ of current inhibition at $\mathrm{pH} 5$ of some of the GMQ derivatives, inhibition curves were recorded for the 5-phenylpyridine 5a and its two derivatives $\mathbf{5 b}$ and $\mathbf{5 c}$, on ASIC1a and ASIC3 (Fig. 5). This showed that in both ASIC1a and ASIC3, these three compounds inhibit currents at lower concentrations than does GMQ. IC $_{50}$ values were $3.3 \pm 0.8 \mathrm{mM}$ (ASIC1a) and $7.4 \pm 1.1 \mathrm{mM}$ (ASIC3) for GMQ, $0.53 \pm 0.07$ and $0.58 \pm 0.11 \mathrm{mM}$ for $\mathbf{5 a}, 0.47 \pm 0.28$ and $0.23 \pm 0.08 \mathrm{mM}$ 
for $\mathbf{5 b}$ and $0.19 \pm 0.01$ and $0.27 \pm 0.04 \mathrm{mM}$ for $\mathbf{5 c}(\mathrm{n}=3-6)$. This up to 20 -fold increase in potency relative to GMQ was significant for $\mathbf{5 c}$ in ASIC1a, and for $\mathbf{5 b}$ and $\mathbf{5 c}$ in ASIC3 $(\mathrm{p}<0.01)$.

\section{Modulation of heteromeric ASIC currents by GMQ derivatives}

In the central nervous system, functional ASICs are mostly ASIC1a homomers, ASIC1a/2a heteromers and ASIC1a/2b heteromers, while in the peripheral nervous system most functional ASICs are heteromers of various compositions ${ }^{1,7,33}$. To expand our analysis, we determined the effect of selected compounds at a concentration of $1 \mathrm{mM}$ on two different ASIC heteromers, ASIC1a/2a, representative of a central nervous system ASIC, and ASIC2a/3, representative of a peripheral nervous system ASIC. Heteromeric ASICs adapt a flexible stoichiometry that depends on the subunit availability, as shown for ASIC1a/2a ${ }^{5}$. The inclusion of ASIC2a subunits shifts the $\mathrm{pH} 50$ to more acidic values, in the range of pH5.5 - pH6 for both ASIC1a/2a and ASIC2a/3 ${ }^{34,35}$. Measurements addressing the shift in $\mathrm{pH}$ dependence ("gating effect") were therefore carried out at pH5.8, while testing for effects on the maximal current amplitude ("blocking effect") was done at $\mathrm{pH} 4$. An $\mathrm{I}_{\mathrm{pH} 5.8} / \mathrm{I}_{\mathrm{pH} 4.0}$ ratio close to $\sim 0.5$ served as an indication that the investigated currents were indeed mediated by heteromeric channels (Table S3). In ASIC1a/2a, only about half of the tested compounds induced amplitude changes that were significantly different between pH5.8 and 4 and indicated thus a shift of the $\mathrm{pH}$ dependence (Fig. 6a). With GMQ, 4a and $\mathbf{6}$, the $I_{\text {cpd }} / I_{\text {ctrl }}$ ratio was greater at $\mathrm{pH} 5.8$ than at $\mathrm{pH} 4$, indicating therefore an alkaline shift of the $\mathrm{pH}$ dependence of activation, opposed to what we had observed with ASIC1a homomers. The absence of shifts in $\mathrm{pH}$ dependence by many, and the predicted alkaline shift by GMQ, 4a and $\mathbf{6}$ in the ASIC1a/2a heteromers may be explained by the previously documented opposite GMQinduced shifts of the activation $\mathrm{pH}$ dependence in ASIC1a and ASIC2a ${ }^{25}$. In ASIC2a/3 heteromers, several compounds showed a tendency of increased $I_{\text {cpd }} / I_{\text {ctrl }}$ ratios at $\mathrm{pH} 5.8$ over $\mathrm{pH} 4$. These differences were however only significant for $\mathbf{4 a}$ and $\mathbf{8}$ (Fig. 6b). We observed a high cell-to-cell variability of the $I_{\text {cpd }} / I_{\text {ctrl }}$, and also a variability of the $\mathrm{I}_{\mathrm{pH} 5.8} / \mathrm{I}_{\mathrm{pH} 4}$ ratio in the absence of any compound, which indicates differences of the ASIC2a:ASIC3 ratio of these heteromers. Interestingly, the potentiation of the pH5.8induced current by compounds 3a and $\mathbf{4 a}$ was greater in cells with a low $\mathrm{I}_{\mathrm{pH} 5.8} / \mathrm{I}_{\mathrm{pH} 4}$ ratio, thus in cells with an important contribution of ASIC2a (Fig. S2). 
Only few compounds affected the $\mathrm{I}_{\mathrm{pH} 4}$ amplitude in the heteromers, suggesting that the inclusion of ASIC2a may distort the GMQ binding site for blocking. Taken together, heteromers with ASIC2a are generally less modulated by GMQ and its derivatives than the ASIC1a and ASIC3 homomers. Some compounds however exert strong effects on the heteromers. No acidic shifts in $\mathrm{pH}$ dependence were induced in the heteromers, showing that the response of heteromers to GMQ and its derivatives is closer to that of ASIC3 than ASIC1a.

\section{Potentiation of ASICla and ASIC heteromer currents by GMQ derivatives at low concentrations}

Whereas most of the tested GMQ derivatives led to an inhibition of both ASIC1a and ASIC3 pH5induced currents at sub-millimolar concentrations, we observed that at a lower concentration $(<100$ $\mu \mathrm{M})$, compounds of several clusters induced an unexpected potentiation of the pH6.6-induced ASIC1a current (Fig. 7). The quinazoline GMQ did not potentiate ASIC1a currents, whereas its quinoline analogs $\mathbf{3 a}$ and $\mathbf{3 b}$ showed a significant potentiation (of $\sim 50 \%$ ) of the current at $30 \mu \mathrm{M}$. This potentiation effect was even observed at $10 \mu \mathrm{M}$ with the 4- and 6-phenylpyridines 6 and $4 \mathbf{a}$, whereas the 5phenylpyridine 5a induced only a very small potentiation at $10 \mu \mathrm{M}$ and none at $30 \mu \mathrm{M}$. The ASIC inhibitor amiloride did not induce any potentiation at 10 and $30 \mu \mathrm{M}$. A possible potentiation by amiloride might be hidden by its substantial pore block at this concentration ${ }^{15,19}$. At lower concentrations that do not inhibit ASIC1a, amiloride had however no effect on ASIC1a currents (Fig. 7). No such effect was observed with any of the tested compounds at 10-30 $\mu \mathrm{M}$ on ASIC3 (Fig. S3a) or ASIC1a/2a (at pH5.8, Fig. S3b). In ASIC2a/3, GMQ induced at these concentrations a small inhibition, and 5a induced a strong potentiation (Fig. S3c). A further analysis of the potentiation effects on ASIC1a was then carried out with compound 6. The $\mathrm{EC}_{50}$ for increasing the current amplitude was $9.4 \pm 5.4 \mu \mathrm{M}$ (error of the fit, $\mathrm{n}=3-8$ ), while the $\mathrm{IC}_{50}$ of inhibition was $93.4 \pm 0.4 \mu \mathrm{M}(\mathrm{n}=4$, Fig. $8 \mathrm{a}$ ). To test whether the current increase was due to a change in $\mathrm{pH}$ dependence, we measured the $\mathrm{pH}$ dependence of activation and of SSD of ASIC1 a and ASIC3 in the absence and the presence of $30 \mu \mathrm{M}$

6. Compound $\mathbf{6}$ did not affect the $\mathrm{pH}_{50}$ values of activation or desensitization, it changed however the steepness of the $\mathrm{pH}$ dependence curves, rendering the ASIC1a activation curve steeper, and both curves 
of ASIC3 less steep (Fig. 8b-c, Table S4). These changes can only partially explain the observed current increase in ASIC1a. We observed however also a slowing of the desensitization time course of ASIC1a (Fig. 8d). This indicates that the transition into the desensitized state is slowed in the presence of 30 $\mu \mathrm{M}$ compound 6, which will increase the current amplitude, and will contribute to the ASIC1a current potentiation. The kinetics of current appearance showed no apparent change in the presence of compound 6 (Fig. 8d). In our whole-cell experiments, the kinetics of current appearance are however limited by the speed of perfusion change (Methods), which is slower than channel opening ${ }^{36}$, and we can therefore not draw conclusions about the opening kinetics.

Potentiating ASIC currents may be of interest in some instances, for example for increasing synaptic transmission or the induction of LTP ${ }^{7}$. For such applications, GMQ derivatives with only a small blocking effect, such as those of cluster \#2, would be more suitable than compound $\mathbf{6}$.

The biphasic effect of GMQ on ASIC $3^{24,25}$, and of these compounds on ASIC1a currents likely reflects binding to at least two binding sites, a modulatory and an inhibitory site, with different affinities, as previously suggested ${ }^{25,37}$. We have shown that the inhibition of ASIC1a and ASIC3 currents at pH5 by GMQ was due to a pore block, since it decreased unitary current amplitudes and was affected by a mutation in the pore ${ }^{25}$. Based on molecular docking, site-directed mutagenesis and the measurement of GMQ-induced current at $\mathrm{pH} 7.4$, a detailed description of a GMQ binding site in the palm domain of ASIC3 has been provided ${ }^{38}$, which is therefore a strong candidate for the "gating" binding site. A later analysis showed however that mutation of some residues suggested to be central for GMQ binding in the palm suppressed the GMQ-induced shift of the $\mathrm{pH}$ dependence of SSD, but not that of activation ${ }^{25}$. This suggested that either the individual mutations of palm residues cannot sufficiently disrupt GMQ binding, or that these mutations do not affect the GMQ binding itself, but rather the consequences of binding. The low apparent affinity of GMQ precludes a more precise analysis of its binding site. The GMQ derivatives with higher affinity than GMQ will allow studies that aim at identifying the precise GMQ binding site(s). This will help elucidating the activation mechanism of ASICs, and the development of more potent and specific ASIC modulators.

\section{Methods}




\section{Recombinant expression of ASICs in Chinese hamster ovary (CHO) cells}

CHO cell lines stably expressing human ASIC1a or rat ASIC3 had been established as indicated elsewhere ${ }^{39}$, based on the human ASIC1a clone of the Corey laboratory ${ }^{32}$, and the rat ASIC3 clone of the Lazdunski laboratory ${ }^{30}$. Heteromeric ASICs were expressed by transient co-transfection of the above mentioned ASIC1a and ASIC3 cDNAs together with ASIC2a cDNA. The protein sequence of the human ASIC2a clone used here ${ }^{32}$, is $99 \%$ identical with the rat ASIC2a clone. Transfections were carried out with ASIC clones in the peak8 expression vector (Edge Biosystems, Gaithersburg, MD), together with a green fluorescent protein construct, by using Roti-Fect (Carl Roth, Karlsruhe, Germany). The cDNA ratios for the transfections was 1:1 for ASIC1a/ASIC2a heteromers and 2:1 for ASIC2a/ASIC3 heteromers. The measured IpH5.8/IpH4 ratios confirmed that the expressed channels were heteromeric (Table S3).

\section{Electrophysiology}

Electrophysiological measurements were carried out in the whole-cell patch-clamp mode at $-60 \mathrm{mV}$ with an EPC9 or EPC10 amplifier (HEKA Electronics, Lambrecht, Germany) and data acquisition was done with the Patchmaster software. Data were acquired at a sampling rate of $1 \mathrm{~ms}$ and filtered at 3 kHz. Pipettes were pulled from borosilicate glass (World Precision Instruments, Stevenage, UK). They had resistances of 2-5 $\mathrm{M} \Omega$ when filled with the pipette solution. Series resistance compensation was set to $70-95 \%$. The extracellular recording solution contained, in $\mathrm{mM}, \mathrm{NaCl} 140, \mathrm{KCl} 4, \mathrm{CaCl}_{2} 2, \mathrm{MgCl}_{2}$ 1, 4-morpholineethanesulfonic acid (MES) 10, HEPES 10, Glucose 10. The $\mathrm{pH}$ was adjusted to the desired value with $\mathrm{NaOH}$. Conditioning solution (applied between acidifications) had a $\mathrm{pH}$ of 7.4. Fast solution changes were carried out with a MPRE8 perfusion head (Cell Micro-Controls, Norfolk, VA) and computer-controlled electrovalves from either Cell Micro-Controls or Biologic (Claix, France). The speed of the perfusion change at the cells was determined on the sustained component of the ASIC3 current at $\mathrm{pH} 7.0$ by measuring the kinetics of the change in current amplitude when the perfusion solution was changed between the standard extracellular solution containing $140 \mathrm{mM} \mathrm{Na}^{+}$and a solution in which the $\mathrm{Na}^{+}$was replaced by the larger, ASIC-impermeable cation $\mathrm{NMDG}^{+}$. The $(10 \%$ to $90 \%)$ rise time of the solution change was $210 \pm 20 \mathrm{~ms}, \mathrm{n}=6$. The pipette solution contained, in $\mathrm{mM}, \mathrm{K}-$ 
gluconate $90, \mathrm{KCl} 10, \mathrm{NaCl} 10, \mathrm{MgCl}_{2}$ 1, HEPES 60, EGTA 10, and its $\mathrm{pH}$ was adjusted to 7.3 with KOH. Data were analyzed with Fitmaster (HEKA). The compounds were included in the acidic extracellular solution (pH6.6 and $\mathrm{pH} 5$ ), and the $\mathrm{pH}$ of the solution was verified after addition of the compound, and adjusted if necessary.

\section{Analysis and Statistics}

Normalized activation curves ( $\mathrm{pH}$ dependence of peak current amplitudes) were fitted to the Hill equation $\mathrm{I}=\mathrm{I}_{\max } /\left(1+\left(10^{-\mathrm{pH} 50} / 10^{-\mathrm{pH}}\right)^{\mathrm{nH}}\right)$, where $\mathrm{I}_{\max }$ is the maximal current amplitude, $\mathrm{pH}_{50}$ is the value at which the current amplitude is half-maximal and $\mathrm{nH}$ is the Hill coefficient. SSD curves were fitted to an analogous equation to obtain the $\mathrm{pHD}_{50}$ values. Data are presented as mean $\pm \mathrm{SEM}$. To determine significance of differences in multiple comparisons we used two-way ANOVA followed by post-hoc Tukey test. To determine whether the amplitude change by a given compound was significant, we used a paired t-test (Graphpad Prism 7 and Microsoft Excel).

To estimate $\mathrm{pH}_{50}$ shifts from current ratios at $\mathrm{pH} 6.6$, currents were expressed as a function of $\mathrm{pH} 50$ and $\mathrm{nH}$ by using the Hill equation, using $\mathrm{nH}$ values for the control situation previously measured in control conditions for each of the two subtypes, and the $\mathrm{pH} 50$ calculated for each condition from the measured current amplitudes induced by pH6.6 and 5.0 in the absence of compound. The ratio $\mathrm{I}_{(\mathrm{compound}, \mathrm{pH} 6.6)} /$ $\mathrm{I}_{(\mathrm{control}, \mathrm{pH} 6.6)}$ was then calculated based on these equations, and $\mathrm{pH}_{50}$ (compound) was adjusted, while keeping $\mathrm{nH}$ unchanged, until the calculated $\mathrm{I}_{(\mathrm{compound}, \mathrm{pH} 6.6)} / \mathrm{I}_{(\mathrm{control}, \mathrm{pH} 6.6)}$ ratio matched the experimental value of a given compound and ASIC subtype. The shift in $\mathrm{pH}_{50}$ was then calculated as $\mathrm{pH}_{50}$ (compound)- $\mathrm{pH}_{50}$ (control). In three cases with $\mathrm{ASIC} 3$ (marked as open bars in Fig. 4b), the measured $\mathrm{I}_{(\mathrm{compound}, \mathrm{pH} \text { 6.6) }} / \mathrm{I}_{(\mathrm{control}, \mathrm{pH} 6.6)}$ ratio was greater than the theoretically possible value, most likely because the compound also induced a change in the Hill coefficient. For these cases we plot in Fig. 4b the $\Delta \mathrm{pH}_{50}$ corresponding to $95 \%$ of the maximal theoretical $\mathrm{I}_{\text {(compound, } \mathrm{pH} \text {.6) }} / \mathrm{I}_{\text {(control, } \mathrm{pH} \text {.6) }}$ ratio.

\section{Substances}


The compounds were synthesized as described in the Supporting Information. GMQ was purchased from Sigma. Stock solutions of compounds were made in DMSO at $100 \mathrm{mM}$. Final concentrations of DMSO were maximally $1 \%$.

\section{Supporting information}

Three supplemental figures, showing 1) sustained current amplitudes of ASIC3 at various pH values, 2) a correlation of compound effects with relative subunit expression, and 3) current ratios in cells expressing ASIC3 or heteromeric ASICs; 4 supplemental tables, containing 1) pKa values of compounds, $2 \&$ 3) current ratios, 4) pH dependence parameters; a methods section describing in detail the compound synthesis.

\section{Abbreviations}

ASIC, acid-sensing ion channel; $\mathrm{CHO}$, Chinese hamster ovary; $\mathrm{ENaC}$, epithelial $\mathrm{Na}^{+}$channel; GMQ, 2-guanidine-4-methylquinazoline; $\mathrm{I}_{\mathrm{cpd}}$, $\mathrm{pH}$-induced current obtained in the presence of a compound; $\mathrm{I}_{\mathrm{ctrl}}, \mathrm{pH}$-induced current obtained under control conditions; $\mathrm{I}_{\mathrm{GMQ}}, \mathrm{pH}$-induced current in the presence of GMQ; $\mathrm{I}_{\text {peak, }}$ peak current; $\mathrm{I}_{\text {sust, }}$ sustained current; $\mathrm{nH}$, Hill coefficient; $\mathrm{pH}_{50}, \mathrm{pH}$ of half-maximal activation; $\mathrm{pHD}_{50}, \mathrm{pH}$ of half-maximal steady-state desensitization; SSD, steady-state desensitization.

\section{Author contributions}

O.A., V. T. and A.V. performed electrophysiological experiments and data analysis; H.H., M.B. and M.S. synthesized of the compounds; O.A., F.B. and S.K. designed the project, analyzed the data, prepared the figures and wrote the manuscript.

\section{Conflict of interest}

The authors declare no conflict of interest.

\section{Acknowledgements}


This work is supported by the Swiss National Science Foundation grant 31003A_172968 to S.K. The authors thank Laurent Schild, Sabrina Vullo, Miguel van Bemmelen and Zhong Peng for their comments on a previous version of the manuscript, and for many discussions.

\section{References}

1. Kellenberger, S., and Schild, L. (2015) International Union of Basic and Clinical Pharmacology. XCI. Structure, Function, and Pharmacology of Acid-Sensing Ion Channels and the Epithelial $\mathrm{Na}^{+}$Channel, Pharmacol Rev 67, 1-35.

2. Yang, L., and Palmer, L. G. (2014) Ion conduction and selectivity in acid-sensing ion channel $1, J$ Gen Physiol 144, 245-255.

3. Deval, E., Noel, J., Lay, N., Alloui, A., Diochot, S., Friend, V., Jodar, M., Lazdunski, M., and Lingueglia, E. (2008) ASIC3, a sensor of acidic and primary inflammatory pain, EMBO J 27, 3047-3055.

4. Vukicevic, M., and Kellenberger, S. (2004) Modulatory effects of acid-sensing ion channels on action potential generation in hippocampal neurons, Am J Physiol Cell Physiol 287, C682-690.

5. Bartoi, T., Augustinowski, K., Polleichtner, G., Grunder, S., and Ulbrich, M. H. (2014) Acid-sensing ion channel (ASIC) 1a/2a heteromers have a flexible 2:1/1:2 stoichiometry, Proc Natl Acad Sci US A 111, 8281-8286.

6. Baconguis, I., Bohlen, C. J., Goehring, A., Julius, D., and Gouaux, E. (2014) X-ray structure of Acidsensing ion channel 1-snake toxin complex reveals open state of a $\mathrm{Na}^{+}$-selective channel, Cell $156,717-729$.

7. Wemmie, J. A., Taugher, R. J., and Kreple, C. J. (2013) Acid-sensing ion channels in pain and disease, Nature reviews. Neuroscience 14, 461-471.

8. Xiong, Z. G., Zhu, X. M., Chu, X. P., Minami, M., Hey, J., Wei, W. L., MacDonald, J. F., Wemmie, J. A., Price, M. P., Welsh, M. J., and Simon, R. P. (2004) Neuroprotection in ischemia: Blocking calcium-permeable acid- sensing ion channels, Cell 118, 687-698.

9. Kreple, C. J., Lu, Y., Taugher, R. J., Schwager-Gutman, A. L., Du, J., Stump, M., Wang, Y., Ghobbeh, A., Fan, R., Cosme, C. V., Sowers, L. P., Welsh, M. J., Radley, J. J., LaLumiere, R. T., and Wemmie, J. A. (2014) Acid-sensing ion channels contribute to synaptic transmission and inhibit cocaine-evoked plasticity, Nat Neurosci 17, 1083-1091.

10. Du, J., Reznikov, L. R., Price, M. P., Zha, X. M., Lu, Y., Moninger, T. O., Wemmie, J. A., and Welsh, M. J. (2014) Protons are a neurotransmitter that regulates synaptic plasticity in the lateral amygdala, Proc Natl Acad Sci U S A 111, 8961-8966.

11. Ziemann, A. E., Allen, J. E., Dahdaleh, N. S., Drebot, II, Coryell, M. W., Wunsch, A. M., Lynch, C. M., Faraci, F. M., Howard, M. A., 3rd, Welsh, M. J., and Wemmie, J. A. (2009) The 
amygdala is a chemosensor that detects carbon dioxide and acidosis to elicit fear behavior, Cell 139, 1012-1021.

12. Chen, C. C., and Wong, C. W. (2013) Neurosensory mechanotransduction through acid-sensing ion channels, Journal of cellular and molecular medicine 17, 337-349.

13. Omerbasic, D., Schuhmacher, L. N., Bernal Sierra, Y. A., Smith, E. S., and Lewin, G. R. (2015) ASICs and mammalian mechanoreceptor function, Neuropharmacol 94, 80-86.

14. Liu, M. G., Li, H. S., Li, W. G., Wu, Y. J., Deng, S. N., Huang, C., Maximyuk, O., Sukach, V., Krishtal, O., Zhu, M. X., and Xu, T. L. (2016) Acid-sensing ion channel 1a contributes to hippocampal LTP inducibility through multiple mechanisms, Sci Rep 6.

15. Schild, L., Schneeberger, E., Gautschi, I., and Firsov, D. (1997) Identification of amino acid residues in the alpha, beta, and gamma subunits of the epithelial sodium channel $(\mathrm{ENaC})$ involved in amiloride block and ion permeation, J Gen Physiol 109, 15-26.

16. Kellenberger, S., Gautschi, I., and Schild, L. (2003) Mutations in the epithelial $\mathrm{Na}^{+}$channel ENaC outer pore disrupt amiloride block by increasing its dissociation rate, Mol. Pharmacol. 64, 848856.

17. Kleyman, T. R., and Cragoe, E. J. (1988) Amiloride and Its Analogs as Tools in the Study of IonTransport, J Membrane Biol 105, 1-21.

18. Baron, A., and Lingueglia, E. (2015) Pharmacology of acid-sensing ion channels - Physiological and therapeutical perspectives, Neuropharmacology 94, 19-35.

19. Schmidt, A., Rossetti, G., Joussen, S., and Grunder, S. (2017) Diminazene Is a Slow Pore Blocker of Acid-Sensing Ion Channel 1a (ASIC1a), Mol Pharmacol.

20. Chen, X., Qiu, L., Li, M., Durrnagel, S., Orser, B. A., Xiong, Z. G., and MacDonald, J. F. (2010) Diarylamidines: high potency inhibitors of acid-sensing ion channels, Neuropharmacology 58 , 1045-1053.

21. Rash, L. D. (2017) Acid-Sensing Ion Channel Pharmacology, Past, Present, and Future, Adv Pharmacol 79, 35-66.

22. Lee, J. Y. P., Saez, N. J., Cristofori-Armstrong, B., Anangi, R., King, G. F., Smith, M. T., and Rash, L. D. (2017) Inhibition of acid-sensing ion channels by diminazene and APETx2 evoke partial and highly variable antihyperalgesia in a rat model of inflammatory pain, $\mathrm{Br} J$ Pharmacol.

23. Baron, A., Diochot, S., Salinas, M., Deval, E., Noel, J., and Lingueglia, E. (2013) Venom toxins in the exploration of molecular, physiological and pathophysiological functions of acid-sensing ion channels, Toxicon 75, 187-204.

24. Yu, Y., Chen, Z., Li, W. G., Cao, H., Feng, E. G., Yu, F., Liu, H., Jiang, H., and Xu, T. L. (2010) A nonproton ligand sensor in the acid-sensing ion channel, Neuron 68, 61-72.

25. Alijevic, O., and Kellenberger, S. (2012) Subtype-specific modulation of acid-sensing ion channel (ASIC) function by 2-guanidine-4-methylquinazoline, J Biol Chem 287, 36059-36070. 
26. Yang, X. N., Niu, Y. Y., Liu, Y., Yang, Y., Wang, J., Cheng, X. Y., Liang, H., Wang, H. S., Hu, Y. M., Lu, X. Y., Zhu, M. X., Xu, T. L., Tian, Y., and Yu, Y. (2017) The nonproton ligand of acidsensing ion channel 3 activates mollusk-specific $\mathrm{FaNaC}$ channels via a mechanism independent of the native FMRFamide peptide, $J$ Biol Chem 292, 21662-21675.

27. Li, W. G., Yu, Y., Zhang, Z. D., Cao, H., and Xu, T. L. (2011) ASIC3 channels integrate agmatine and multiple inflammatory signals through the nonproton ligand sensing domain, Mol Pain 6, 88.

28. Li, W. G., Yu, Y., Huang, C., Cao, H., and Xu, T. L. (2011) The nonproton ligand sensing domain is required for paradoxical stimulation of ASIC3 channels by amiloride, $J$ Biol Chem 286, $42635-42646$.

29. Besson, T., Lingueglia, E., and Salinas, M. (2017) Pharmacological modulation of Acid-Sensing Ion Channels $1 \mathrm{a}$ and 3 by amiloride and 2-guanidine-4-methylquinazoline (GMQ), Neuropharmacology 125, 429-440.

30. Waldmann, R., Bassilana, F., Deweille, J., Champigny, G., Heurteaux, C., and Lazdunski, M. (1997) Molecular cloning of a non-inactivating proton-gated $\mathrm{Na}^{+}$channel specific for sensory neurons, J. Biol. Chem. 272, 20975-20978.

31. Waldmann, R., Champigny, G., Bassilana, F., Heurteaux, C., and Lazdunski, M. (1997) A protongated cation channel involved in acid-sensing, Nature 386, 173-177.

32. Garcia-Anoveros, J., Derfler, B., Nevillegolden, J., Hyman, B. T., and Corey, D. P. (1997) BNaC1 and $\mathrm{BNaC} 2$ constitute at new family of human neuronal sodium channels related to degenerins and epithelial sodium channels, Proc. Natl. Acad. Sci. USA 94, 1459-1464.

33. Benson, C. J., Xie, J. H., Wemmie, J. A., Price, M. P., Henss, J. M., Welsh, M. J., and Snyder, P. M. (2002) Heteromultimers of DEG/ENaC subunits form $\mathrm{H}^{+}$-gated channels in mouse sensory neurons, Proc. Natl. Acad. Sci. USA 99, 2338-2343.

34. Hesselager, M., Timmermann, D. B., and Ahring, P. K. (2004) pH dependency and desensitization kinetics of heterologously expressed combinations of acid-sensing ion channel subunits, J. Biol. Chem. 279, 11006-11015.

35. Joeres, N., Augustinowski, K., Neuhof, A., Assmann, M., and Grunder, S. (2016) Functional and pharmacological characterization of two different ASIC1a/2a heteromers reveals their sensitivity to the spider toxin PcTx1, Sci Rep 6, 27647.

36. Bassler, E. L., Ngo-Anh, T. J., Geisler, H. S., Ruppersberg, J. P., and Grunder, S. (2001) Molecular and functional characterization of acid-sensing ion channel (ASIC) 1b, J. Biol. Chem. 276, 33782-33787.

37. Smith, R. N., and Gonzales, E. B. (2014) Protons and Psalmotoxin-1 reveal nonproton ligand stimulatory sites in chicken acid-sensing ion channel: Implication for simultaneous modulation in ASICs, Channels (Austin) 8, 49-61. 
38. Yu, Y., Li, W. G., Chen, Z., Cao, H., Yang, H., Jiang, H., and Xu, T. L. (2011) Atomic level characterization of the nonproton ligand-sensing domain of ASIC3 channels, J Biol Chem 286, 24996-25006.

39. Poirot, O., Berta, T., Decosterd, I., and Kellenberger, S. (2006) Distinct ASIC currents are expressed in rat putative nociceptors and are modulated by nerve injury, $J$ Physiol 576, 215-234. 


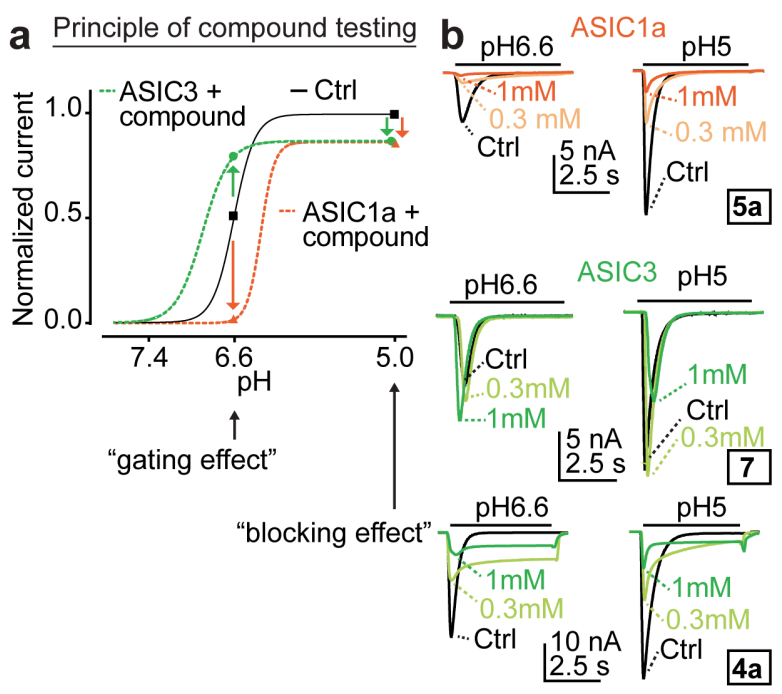

Figure 1. Principle of compound testing. a, Hypothetical $\mathrm{pH}$ dependence of activation is shown for ASIC1a and ASIC3 in the absence of a test compound (solid black line), and in the presence of a compound with similar properties as GMQ on ASIC3 (green dashed line) and ASIC1a (orange dashed line), showing the change in $\mathrm{pH}$ dependence and maximal current amplitude. The arrows indicate the changes in current amplitude measured at pH6.6 ("gating effect") and pH5 ("blocking effect"). b, Traces of a representative experiment with compound 5a on ASIC1a (top) and compounds 7 (center) and $\mathbf{4 a}$ (bottom) on ASIC3 at concentrations of 0.3 and $1 \mathrm{mM}$. ASICs were expressed in CHO cells and their currents were measured with whole-cell patch-clamp. 


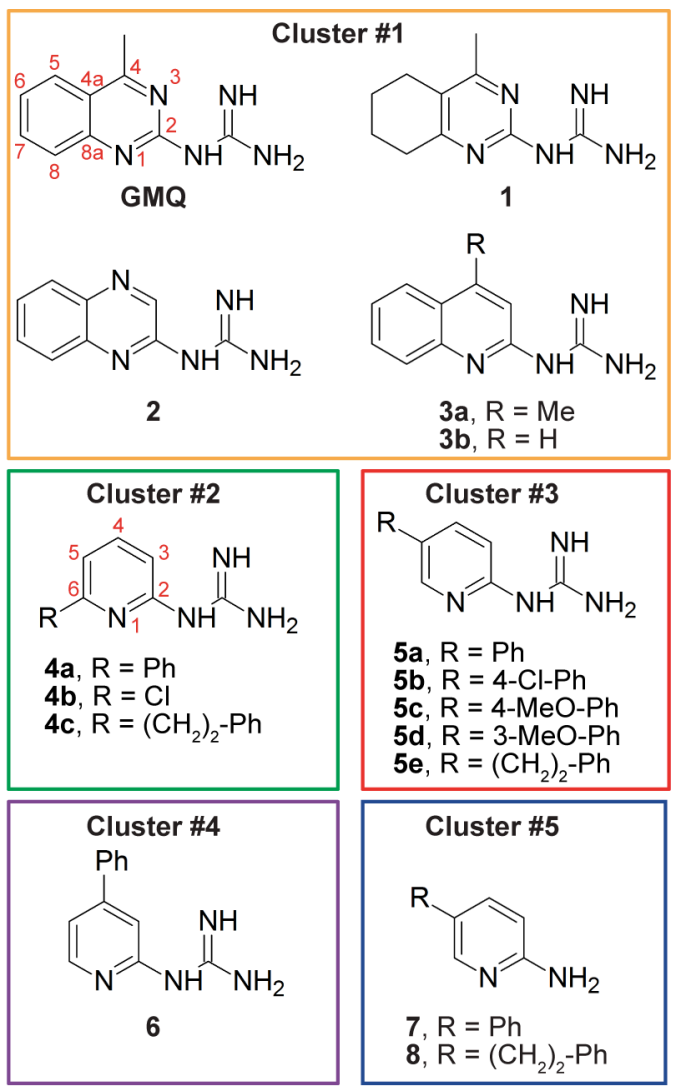

Figure 2. GMQ derivatives tested. Structures of the different compounds tested in this study are shown, and the clusters are identified. 

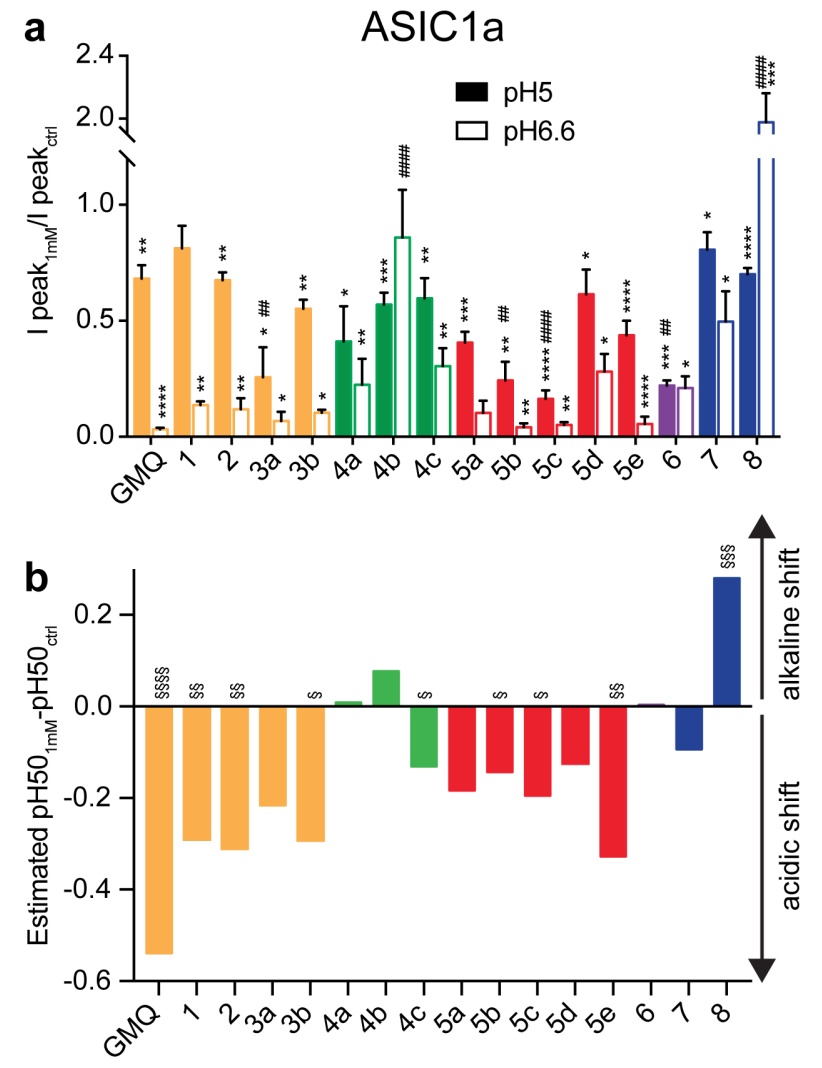

Figure 3. Functional analysis of GMQ derivatives on ASIC1a. a, Bar graph indicating the peak current amplitudes induced with pH5 (filled bars) or pH6.6 (open bars), in the presence of $1 \mathrm{mM}$ test compound, normalized to the current amplitude measured at the same $\mathrm{pH}$ in the absence of the compound. b, Shift of the $\mathrm{pH}$ dependence of activation by different compounds, estimated from comparison of the $I_{\mathrm{cpd}} / I_{\mathrm{ctrl}}$ ratios at $\mathrm{pH} 6.6$ and $\mathrm{pH} 5$ (see Methods). Significance indications are $\mathrm{p}<0.05,0.01,0.001$ and 0.0001 , respectively for $1,2,3$, or 4 symbols, in a, * inhibition at $\mathrm{pH} 5$ or change in amplitude at $\mathrm{pH} 6.6$; \# effect of compound different from GMQ effect; in $\mathrm{b}, \S$, difference between the $\mathrm{I}_{\mathrm{cpd}} / \mathrm{I}_{\mathrm{ctrl}}$ ratio at $\mathrm{pH} 5$ and $\mathrm{pH} 6.6$ for a given compound $(n=3-10)$. 


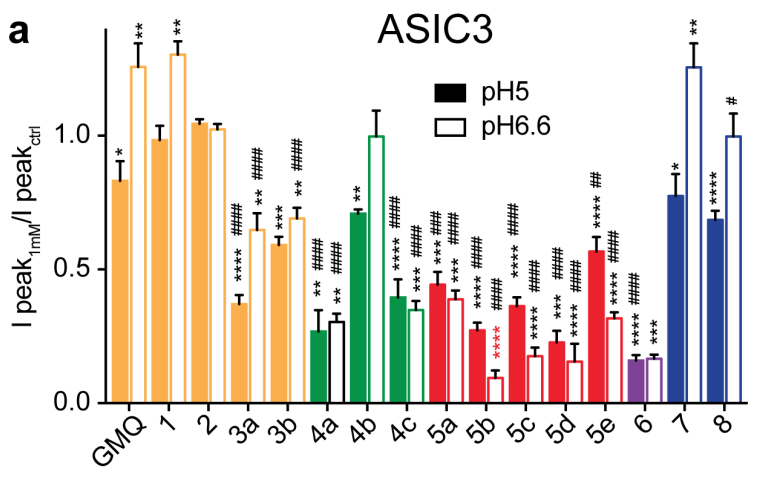

C
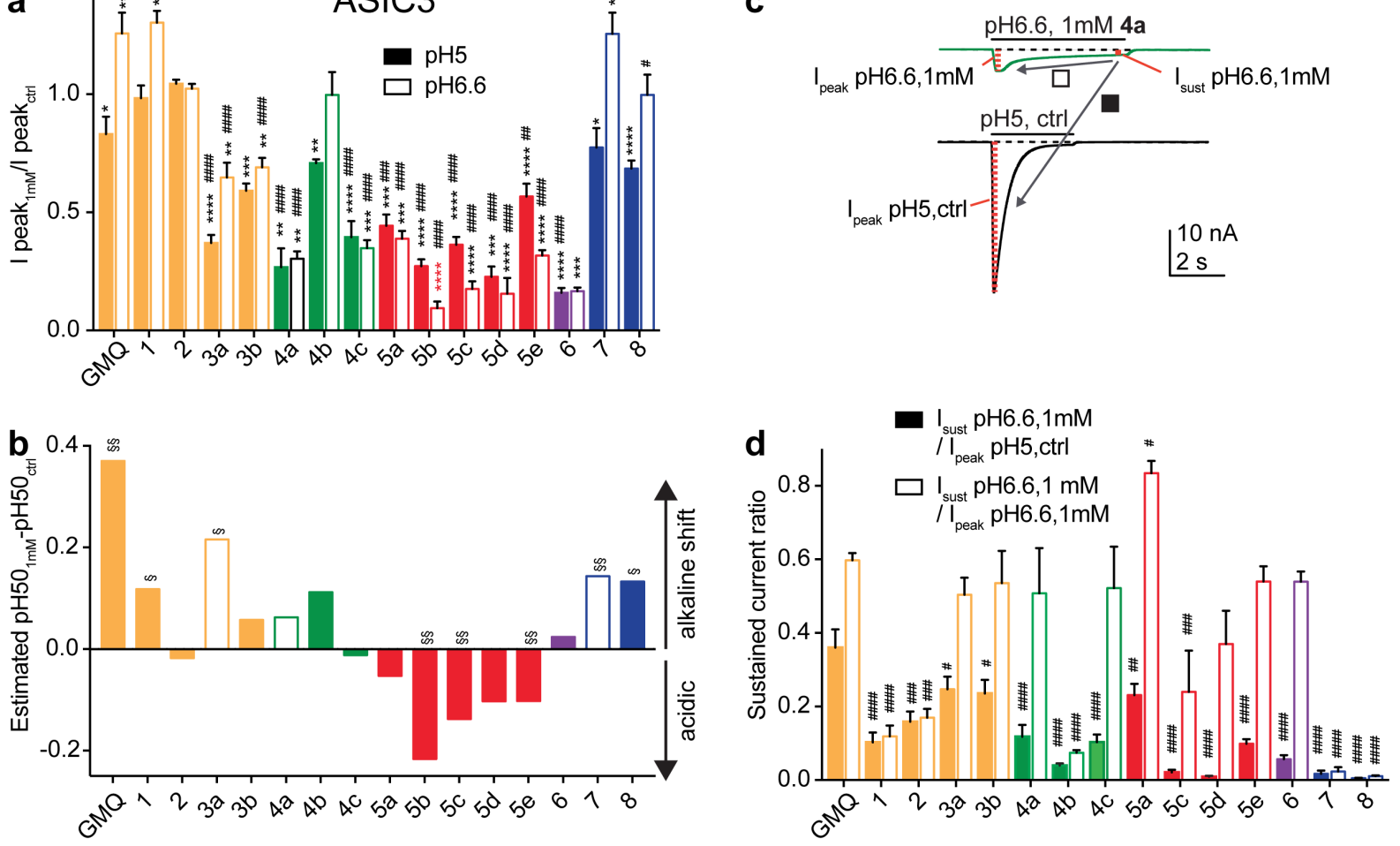

Figure 4. Functional analysis of GMQ derivatives on ASIC3. a, Bar graph indicating the peak current amplitudes induced with pH5 (filled bars) or pH6.6 (open bars), in the presence of $1 \mathrm{mM}$ test compound, normalized to the current amplitude measured at the same $\mathrm{pH}$ in the absence of the compound. $\mathrm{b}$, Shift of the $\mathrm{pH}$ dependence of activation by different compounds, estimated from comparison of the $I_{\mathrm{cpd}} / I_{\mathrm{ctrl}}$ ratios at pH6.6 and pH5 (see Methods). The open bars represent values calculated in a different way (Methods). c, Representative current traces of ASIC3 obtained either by pH6.6 together with $1 \mathrm{mM}$ of the compound 4a (top), or by a control application of $\mathrm{pH} 5.0$ (bottom) from the same cell, to illustrate the two ways of $I_{\text {sust }}$ normalization. The dotted red vertical lines represent the different amplitudes. The arrows indicate the ratio represented in $\mathrm{d}$ by the open bars $\left(\mathrm{I}_{\text {sust }} \mathrm{pH} 6.6,1 \mathrm{mM} / \mathrm{I}_{\text {peak }} \mathrm{pH} 6.6,1 \mathrm{mM}\right)$ and the filled bars $\left(\mathrm{I}_{\text {sust }} \mathrm{pH} 6.6,1 \mathrm{mM} / \mathrm{I}_{\text {peak }} \mathrm{pH} 5.0, \mathrm{ctrl}\right) . \mathrm{d}$, Bar graph plotting the sustained current amplitude induced with $1 \mathrm{mM}$ test compound at pH6.6, normalized either to the peak current induced by $\mathrm{pH} 5 \mathrm{in}$ absence of the compound (filled bars) or the pH6.6-induced peak current in the presence of the compound (open bars; "shape"). Compounds $\mathbf{5 b}$ and $\mathbf{5 d}$ at $1 \mathrm{mM}$ destabilized the recording when applied for $>5 \mathrm{~s}$. Therefore, the sustained current could not be measured in the presence of $\mathbf{5 b}$ and $\mathbf{5 d}$. Significance indications are $\mathrm{p}<0.05,0.01,0.001$ and 0.0001 , respectively for $1,2,3$, or 4 symbols, in panel a, * inhibition at $\mathrm{pH} 5$ or change in amplitude at $\mathrm{pH} 6.6$; \# effect of compound different from GMQ 
effect; in $\mathrm{b}, \S$, difference between the $\mathrm{I}_{\mathrm{cpd}} / \mathrm{I}_{\text {ctrl }}$ ratio at $\mathrm{pH} 5$ and $\mathrm{pH} 6.6$ for a given compound; in $\mathrm{d}$, \#, $\mathrm{I}_{\text {sust }}$ $/ \mathrm{I}_{\text {peak }}$ ratio different from that of GMQ; $\mathrm{n}=3-10$. 

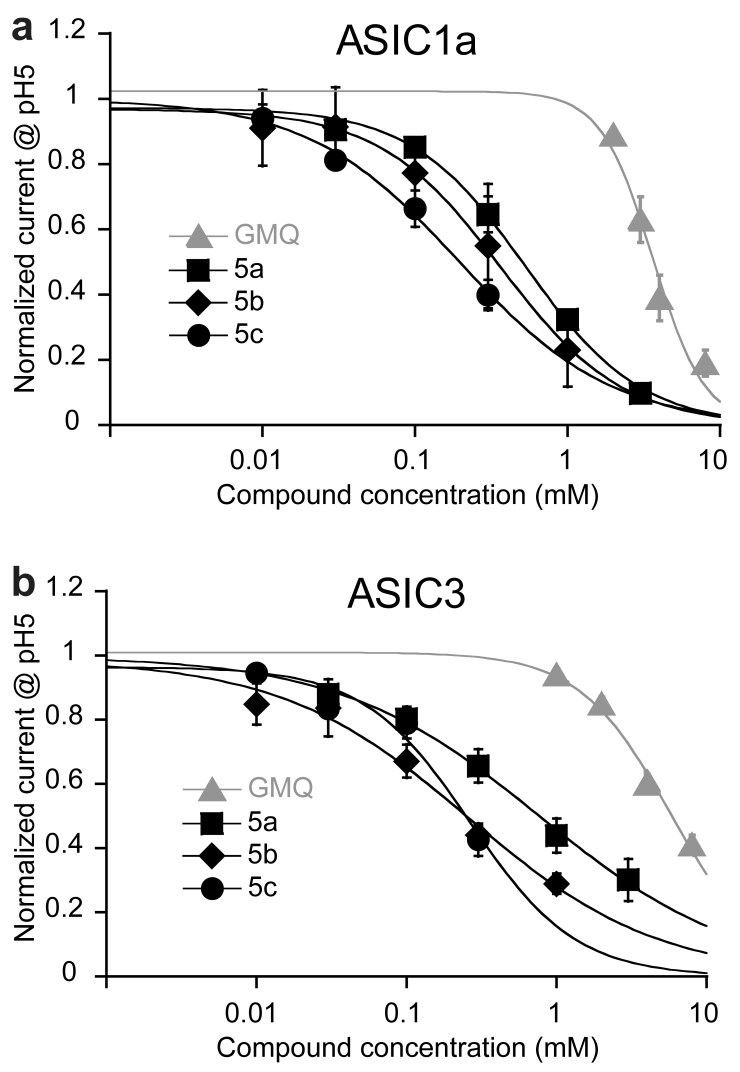

Figure 5. Inhibition curves by selected compounds. The indicated compounds were included at different concentrations in the stimulation solution of $\mathrm{pH} 5$. Current amplitudes measured with compound were normalized to the amplitude obtained in the absence of the modulator. Data are from 3-6 experiments per compound, shown for ASIC1a (a) and ASIC3 (b). Inhibition curves were fitted to a Hill equation, and $\mathrm{IC}_{50}$ values are presented in the text. 

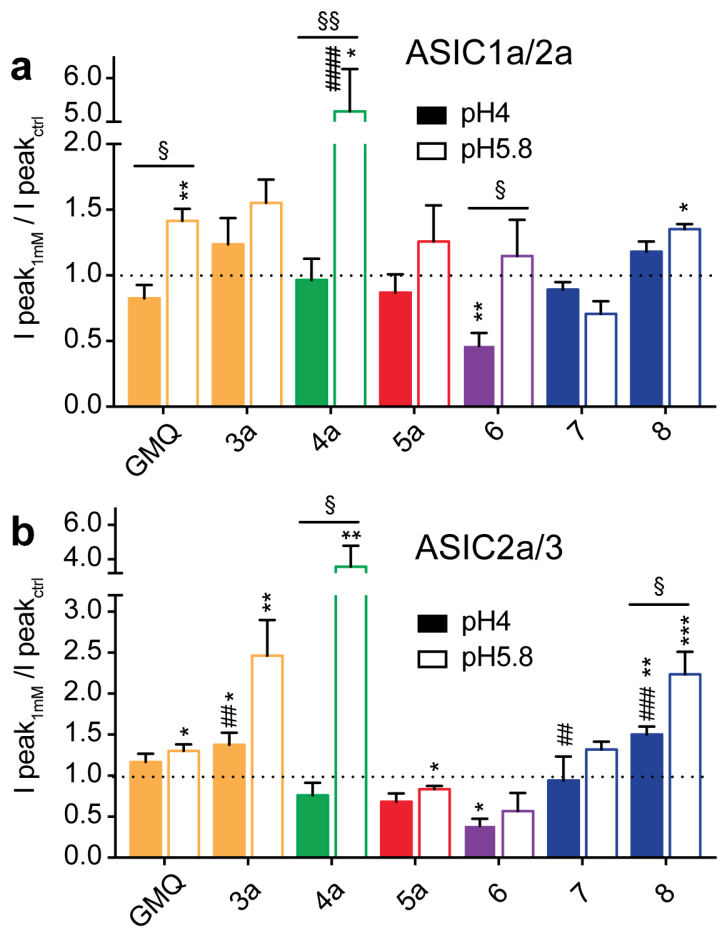

Figure 6. Functional analysis of GMQ derivatives on ASIC1a/2a and ASIC2a/3. a, b, Bar graphs indicating the peak current amplitudes induced with pH4 (filled bars) or pH5.8 (open bars), in the presence of $1 \mathrm{mM}$ test compound, normalized to the current amplitude measured at the same $\mathrm{pH}$ in the absence of the compound, for ASIC1a/2a heteromers (a) and ASIC2a/3 heteromers (b). Significance indications are $\mathrm{p}<0.05,0.01,0.001$ and 0.0001 , respectively for $1,2,3$, or 4 symbols; *, change in amplitude by the compound at $\mathrm{pH} 4$ or 5.8 ; \#, effect of compound different from GMQ effect; $\S$, difference between the $\mathrm{I}_{\mathrm{cpd}} / \mathrm{I}_{\mathrm{ctrl}}$ ratio at $\mathrm{pH} 4$ and $\mathrm{pH} 5.8$ for a given compound $(\mathrm{n}=3-7)$. 


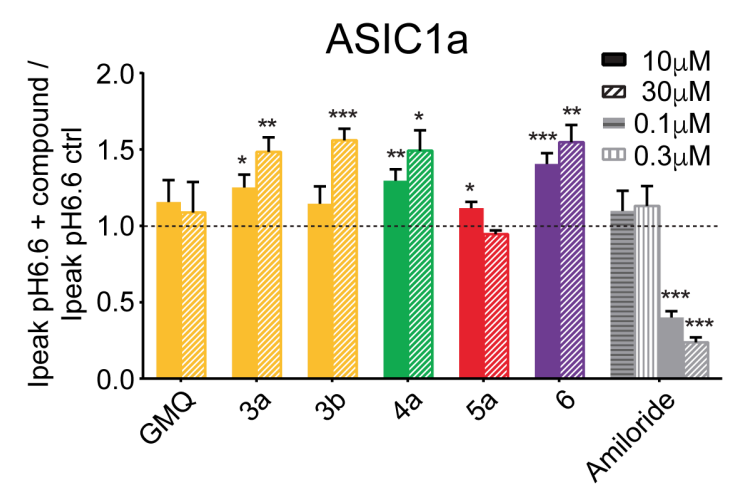

Figure 7. Potentiation of ASIC1 a currents by selected GMQ derivatives. ASIC1a currents were induced by pH6.6 with or without test compound. The indicated concentrations of the test compounds were included in the pH6.6 solution. The current amplitude obtained in the presence of a test compound was normalized to the amplitude obtained in its absence. Data were obtained from 3-7 cells per condition *, $\mathrm{p}<0.05, * *, \mathrm{p}<0.01, * * *, \mathrm{p}<0.001$ between test and control condition, paired t-test. 

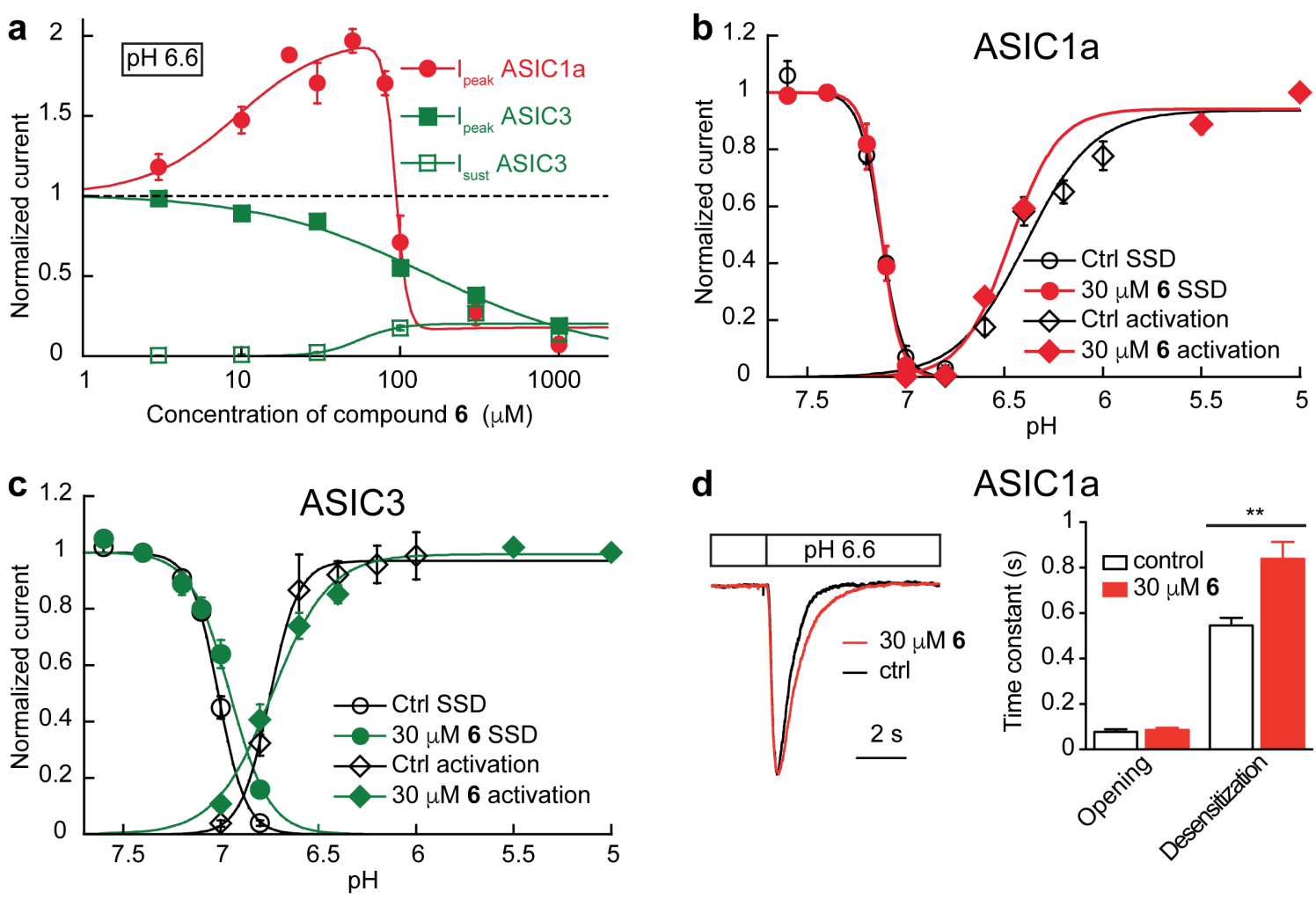

d

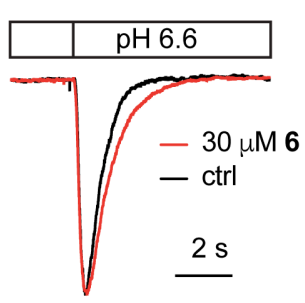

ASIC1a

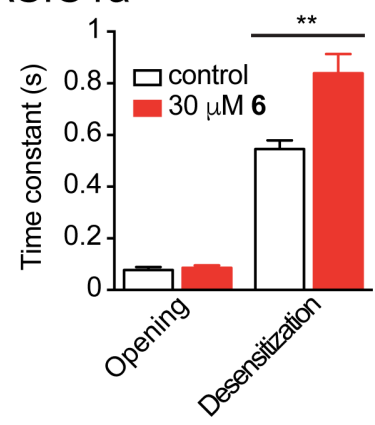

Figure 8. Analysis of ASIC1a current potentiation by compound 6. a, ASIC currents were induced by acidification to $\mathrm{pH} 6.6$ either under control conditions, or with increasing concentrations of compound 6 included in the pH6.6 solution and were normalized to the pH6.6-induced peak current amplitude in the absence of the compound. The concentration-dependent inhibition of ASIC 3 currents (green, solid squares), the appearance of a sustained ASIC3 current (open green squares), and the biphasic effect on ASIC1a (red circles) are shown. The data were fitted to Hill equations; for ASIC1a, a combined equation was used. Fit parameters for ASIC1a are presented in the text. ASIC3 peak current inhibition $\mathrm{IC}_{50}=153.7 \pm 20.4 \mu \mathrm{M}, \mathrm{n}=3-6$; ASIC3 sustained current $\left.\mathrm{EC}_{50}=55.2 \pm 29.5 \mu \mathrm{M}, \mathrm{n}=3-6\right) . \mathrm{b}$ and $\mathrm{c}$, Activation and steady-state desensitization curves obtained under control conditions (open symbols) and in the presence of $30 \mu \mathrm{M}$ compound 6 (filled, colored symbols), for ASIC1a (b) and ASIC3 (c). The fit parameters are shown in Table S4. d, Left, representative current traces of ASIC1a induced by pH6.6 in the absence or presence of $30 \mu \mathrm{M} \mathrm{6}$. The traces were normalized to allow comparison of the kinetics. Right, time constants of opening and desensitization (current decay) of pH6.6-induced current in the absence (open bars) or presence (red) of $30 \mu \mathrm{M} \mathrm{6}, \mathrm{n}=8 .{ }^{* *}, \mathrm{p}<0.01$. 


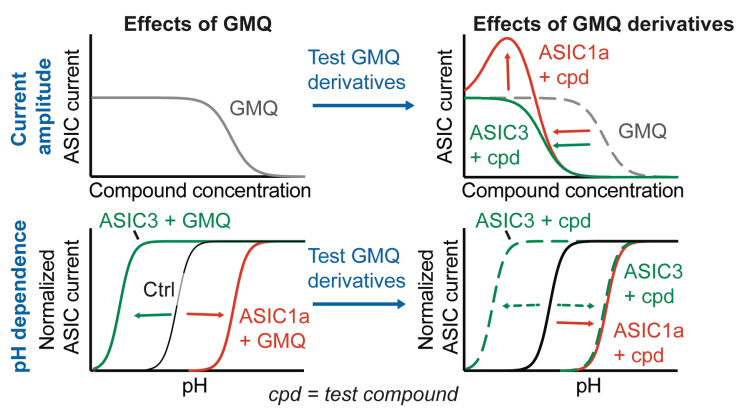

\section{Graphic for Table of contents}

\section{Heteroarylguanidines as Allosteric Modulators of ASIC1a and ASIC3 Channels}

Omar Alijevic, Hassan Hammoud, Anand Vaithia, Viktor Trendafilov, Maud Bollenbach, Martine Schmitt, Frédéric Bihel, Stephan Kellenberger 